\title{
Deformations of the Canonical Commutation Relations and Metric Structures ${ }^{\star}$
}

Francesco D'ANDREA ${ }^{a, c}$, Fedele LIZZI ${ }^{b, c, d}$ and Pierre MARTINETTI ${ }^{b, c}$

a) Dipartimento di Matematica e Applicazioni, Università di Napoli Federico II, Italy E-mail: francesco.dandrea@unina.it

b) Dipartimento di Fisica, Università di Napoli Federico II, Italy E-mail: lizzi@na.infn.it,martinetti.pierre@gmail.com

c) I.N.F.N. - Sezione di Napoli, Italy

d) Departament de Estructura i Constituents de la Matèria, Institut de Ciéncies del Cosmos, Universitat de Barcelona, Spain

Received March 02, 2014, in final form June 01, 2014; Published online June 10, 2014

http://dx.doi.org/10.3842/SIGMA.2014.062

\begin{abstract}
Using Connes distance formula in noncommutative geometry, it is possible to retrieve the Euclidean distance from the canonical commutation relations of quantum mechanics. In this note, we study modifications of the distance induced by a deformation of the position-momentum commutation relations. We first consider the deformation coming from a cut-off in momentum space, then the one obtained by replacing the usual derivative on the real line with the $h$ - and $q$-derivatives, respectively. In these various examples, some points turn out to be at infinite distance. We then show (on both the real line and the circle) how to approximate points by extended distributions that remain at finite distance. On the circle, this provides an explicit example of computation of the Wasserstein distance.
\end{abstract}

Key words: noncommutative geometry; Heisenberg relations; spectral distance

2010 Mathematics Subject Classification: 58B34; 46L87

\section{Introduction}

In this note we study metric structures induced by a deformation of the canonical commutation relations of quantum mechanics. As explained by Connes and Marcolli in [9, Section 10.1], it is precisely the lack of commutativity between the coordinate and the translation operators which permits to retrieve the usual distance from a purely operator-algebraic setting. Specifically, by using the distance formula in noncommutative geometry [8] (see (2) below), one is able to extract the Euclidean distance on $\mathbb{R}^{d}, d \geq 1$, from the canonical commutation relations (CCR)

$$
\begin{aligned}
& {\left[\boldsymbol{x}^{\mu}, \boldsymbol{x}^{\nu}\right]=0, \quad\left[\boldsymbol{p}_{\mu}, \boldsymbol{p}_{\nu}\right]=0,} \\
& {\left[\boldsymbol{x}^{\mu}, \boldsymbol{p}_{\nu}\right]=i \delta_{\nu}^{\mu}}
\end{aligned}
$$

where $\mu, \nu=1, \ldots, d$, we take $\hbar=1$ and $\delta_{\nu}^{\mu}$ is the Kronecker symbol. From this point of view, the CCR encode the metric structure of the classical $\boldsymbol{x}$-space. Let us stress that here by metric structure we mean a distance, not a metric tensor.

Several considerations, mostly inspired by quantum gravity, suggest that space (or spacetime) manifests its quantum nature at very small scale. One of the most elaborated theory of quantized spacetime is loop quantum gravity, where geometrical quantities such as length, area and volume

${ }^{\star}$ This paper is a contribution to the Special Issue on Deformations of Space-Time and its Symmetries. The full collection is available at http://www.emis.de/journals/SIGMA/space-time.html 
are encoded by operators with discrete spectra [32], and are in this sense "quantized". However, the idea that sharp localization should become critical at very small length scales has been around for a long time [5] (see also [26] for an historical account). Later on, it has been shown that gravity-induced uncertainty relations of Heisenberg type prevent the measurement of position with arbitrary precision. Models of quantum spacetime have been developed on this base, starting with a noncommutativity of the coordinate operators $\boldsymbol{x}^{\mu}, \boldsymbol{x}^{\nu}$ (see [12] for the original paper, and [26] for the recent developments). String theory also indicates a minimal uncertainty in space $[1,14]$, which can be encoded in a generalized uncertainty principle coming from a deformation of the $\boldsymbol{x}-\boldsymbol{p}$ commutation relations [18] ${ }^{1}$. There are also models (e.g. [3]) where both position-position and position-momentum commutation relations are deformed.

In this paper, we focus on a deformation of the $\boldsymbol{x}$ - $\boldsymbol{p}$ relation only. The coordinates $\boldsymbol{x}^{\mu}, \boldsymbol{x}^{\nu}$ still commute (as well as $\boldsymbol{p}^{\mu}, \boldsymbol{p}^{\nu}$ ). From Gelfand-Naimark point of view, this means that topologically we are still dealing with a classical space (the algebra of coordinates is commutative). However, since the distance formula of noncommutative geometry crucially depends on the commutators (1b) (as shown in (6)), a modification of the CCR alters the metric.

One way to deform the CCR, inspired by regularization of renormalizable field theories [4, 20], is through the introduction of a cut-off on momenta. This is discussed in Section 2.

In Section 3 we study other modifications of the CCR, focusing for simplicity on the one dimensional case. We first consider the example where on the r.h.s. of (1b) there is an arbitrary function of $\boldsymbol{x}$, which amounts to equipping the real line with an arbitrary Riemannian metric (Proposition 3.1). Then we consider a commutator linear in $\boldsymbol{p}$ induced by a deformation of the derivative called $h$-derivative (see, e.g., [15]). We prove in Proposition 3.4 that the distance between two points $x$ and $y$ is $|x-y|$ if $x-y \in h \mathbb{Z}$, and is infinite otherwise. A last example is the $q$-derivative (see, e.g., [19]), corresponding to the $q$-deformed relation $\boldsymbol{x p}-q \boldsymbol{p} \boldsymbol{x}=i$ [13]. We prove in Proposition 3.8 that the distance is bounded from above by the "French railway metric". We end the section with few considerations on the metric structure of the momentum space.

The picture which emerges from these various examples is that a deformation of the CCR may alter the metric structure of the line in a fundamental way, such as points at infinite distance (Proposition 2.3). This invites to rethink the notion of point. For instance, we show in Proposition 3.5 how to approximate points of the real line by "fat" points (rectangular distributions) which remain at a finite distance. In Section 4 we compute the distance for such fat points on the circle. Proposition 4.1 has an interest on its own (independent of noncommutative geometry), as an example of optimal transport on the circle.

\section{$2 \quad$ Spectral metric geometry}

This section contains some basics on the metric aspect of noncommutative geometry. We show how, in the Euclidean case, Connes formula allows to retrieve the usual distance from the canonical commutation relations of quantum mechanics. Then, we recall how a minimal distance on the position space may emerge from a cut-off in the momentum space. Finally, we investigate the commutation relation coming from the cutoff.

\subsection{Euclidean distance from the canonical commutation relations}

Let $M$ be an oriented Riemannian manifold without boundary, $\mathcal{A}:=C_{0}^{\infty}(M, \mathbb{R})$ the algebra of real smooth functions vanishing at infinity, $\mathcal{H}:=L^{2}(M, E)$ the Hilbert space of square integrable

\footnotetext{
${ }^{1}$ In these analyses, as well as in our study, not only the algebraic commutation relations are important, but also their realization through operators on a Hilbert space. For instance, a characteristic of [18] is the use of symmetric operators with no selfadjoint extensions.
} 
sections of a vector bundle $E \rightarrow M$, and represent $f \in \mathcal{A}$ on $\mathcal{H}$ by pointwise multiplication. Let $D$ be any self-adjoint (possibly unbounded) operator on $\mathcal{H}$ such that

(i) $[D, f]$ is a bounded operator for any $f \in \mathcal{A}$.

Then for $x, y \in M$, the formula

$$
d_{D}(x, y):=\sup \{f(x)-f(y):\|[D, f]\| \leq 1\}
$$

defines an extended metric on $M$ (a distance, except that the value $+\infty$ is allowed). Here the supremum is over all $f \in \mathcal{A}$ satisfying the side-inequality.

The distance (2) can be extended to arbitrary states $\varphi, \psi$ (positive linear functionals with norm 1) of $C_{0}(M, \mathbb{R})$ :

$$
d_{D}(\varphi, \psi):=\sup \{\varphi(f)-\psi(f):\|[D, f]\| \leq 1\} .
$$

Equation (2) is retrieved by considering pure states (extremal points of the convex space of states) which, by Gelfand theorem, are the evaluation at points of $M$. Formula (3) is exactly the Kantorovich dual formula for the Wasserstein distance of order 1 (see [11] and references therein).

When $M$ is locally compact and complete and $D$ is a Dirac operator, it is well known that (2) coincides with the geodesic distance of $M$. A crucial property of (generalized) Dirac operators is that

(ii) $f(D+i)^{-1}$ is a compact operator for all $f \in \mathcal{A}$.

A triple $(\mathcal{A}, \mathcal{H}, D)$ satisfying (i) and (ii) is called a spectral triple, a notion at the core of Connes' noncommutative geometry [8]. Accordingly, we call the extended metric (2) the spectral distance.

On $\mathbb{R}^{d}, d \geq 1$, we interpret the Euclidean distance as the spectral distance defined by phase-space canonical commutation relations. Indeed, the position and momentum operators $\boldsymbol{x}=\left\{\boldsymbol{x}^{\mu}\right\}$ and $\boldsymbol{p}=\left\{\boldsymbol{p}_{\mu}\right\}, \mu=1, \ldots, d$, of $d$-dimensional quantum mechanics are given, in the space representation, by the operators

$$
\boldsymbol{x}^{\mu}(\psi)(x)=x^{\mu} \psi(x), \quad \boldsymbol{p}_{\mu}(\psi)(x)=-i \frac{\partial}{\partial x^{\mu}} \psi(x),
$$

which are essentially selfadjoint on the domain $\mathcal{S}\left(\mathbb{R}^{d}\right) \subset L^{2}\left(\mathbb{R}^{d}\right)$ (the space of Schwartz functions on $\left.\mathbb{R}^{d}\right)$. Since the operators in (4) are endomorphisms of $\mathcal{S}\left(\mathbb{R}^{d}\right)$, their composition is well defined and the CCR (1) are satisfied (for a recent view on the CCR and its mathematical implications, e.g., different choices of domains, see [16]). Moreover, any $f \in C_{0}^{\infty}\left(\mathbb{R}^{d}\right)$ defines a bounded operator $f(\boldsymbol{x})$ on $L^{2}\left(\mathbb{R}^{d}\right)$ given by pointwise multiplication:

$$
f(\boldsymbol{x}) \psi(x):=f(x) \psi(x) \quad \forall \psi \in L^{2}\left(\mathbb{R}^{d}\right), \quad x \in \mathbb{R}^{d} .
$$

The operator (5) maps the domain of self-adjointness of $\boldsymbol{p}_{\mu}$ into itself: the commutator $\left[\boldsymbol{p}_{\mu}, f(\boldsymbol{x})\right]$ is then well-defined, with closure the bounded operator $-i \partial_{\mu} f(\boldsymbol{x})$. If $D=\sum_{\mu} \gamma^{\mu} \boldsymbol{p}_{\mu}$ is the Dirac operator of $\mathbb{R}^{d}$, one easily checks that (see, e.g., the proof of Proposition 2.1 of [11]):

$$
\|[D, f]\|=\sup _{x \in \mathbb{R}^{d}} \sqrt{\sum_{\mu}\left|\partial_{\mu} f(x)\right|^{2}} .
$$

On the left hand side we have the operator norm on the Hilbert space of spinors, and on the right hand side the sup norm of a function of $x$, i.e. its operator norm as an operator on $L^{2}\left(\mathbb{R}^{d}\right)$. 
Denoting the latter simply by $\|[\boldsymbol{p}, f(\boldsymbol{x})]\|$ (it $i s$ the operator norm of the commutator for $d=1$ ), we can rewrite the spectral distance on $\mathbb{R}^{d}$ as

$$
d_{\boldsymbol{p}}(x, y):=\sup _{f \in C_{0}^{\infty}\left(\mathbb{R}^{d}, \mathbb{R}\right)}\{f(x)-f(y):\|[\boldsymbol{p}, f(\boldsymbol{x})]\| \leq 1\},
$$

which of course for $\boldsymbol{p}$ as in (4), satisfying the CCR, gives the Euclidean distance $|x-y|$. In this sense, the metric structure of the position space is determined by the phase-space commutation relations.

Our aim in this paper is to study how the distance is modified if one takes position and momentum operators that satisfy a deformation of the CCR (1b). In Section 2.2 we consider a regularized momentum operator $\boldsymbol{p}$; then in Section 3 we discuss the CCR associated to a Riemannian metric on $\mathbb{R}$, and study various finite-difference approximations of the derivative.

\subsection{Minimal length by cutting-off the geometry}

If we introduce a cut-off in the 'momentum space', i.e. we replace $D$ in (2) by a bounded operator $D_{\Lambda}$ with norm $\Lambda>0$, condition (ii) is no longer satisfied. Nevertheless, condition (i) is trivially satisfied and equation (2) still defines an extended metric on $M$.

Borrowing the terminology of [8], since the length element " $d s=D^{-1}$ " is no longer an infinitesimal (i.e. $f(D+i)^{-1}$ is no longer compact), it is reasonable to expect that points cannot be taken as close as we want anymore, and a minimum length will appear (from a physical point of view, we cannot probe the space with a resolution better than $\Lambda^{-1}$ ). Indeed, one has the following proposition.

Proposition 2.1 ([10, Proposition 5.1]). Let $D$ be bounded with norm $\Lambda>0$. Then for any $x \neq y$,

$$
d_{D}(x, y) \geq \Lambda^{-1}
$$

i.e. the distance between two points cannot be smaller than the cut-off.

Depending on the case, $d_{D_{\Lambda}}(x, y)$ might be infinite for all $x \neq y$, and this would make the inequality (7) not particularly interesting. A sufficient condition to have points at infinite distance is that the commutant of $D$ in $\mathcal{A}:=C_{0}^{\infty}(M, \mathbb{R})$ is non-trivial, i.e. the set of $f \in \mathcal{A}$ such that $[D, f]=0$ contains non-constant functions.

Proposition 2.2. If there exists a non-constant $f \in \mathcal{A}$ such that $[D, f]=0$, then there are (at least) two points at infinite distance $d_{D}$.

Proof. Since $f$ is not constant, we can find $x, y$ such that $f(x)-f(y) \neq 0$. Let $f_{\lambda}=\lambda f$. One has $[D, f]=0$, hence $\left\|\left[D, f_{\lambda}\right]\right\| \leq 1$ and $d_{D}(x, y) \geq\left|f_{\lambda}(x)-f_{\lambda}(y)\right|=\lambda|f(x)-f(y)|$ for all $\lambda>0$. The inequality holds for all $\lambda$, so $d_{D}(x, y)=\infty$.

A non-trivial commutator however is not necessary to have infinite distance, as it can be seen in the next proposition.

Proposition 2.3 ([10, Proposition 5.4]). Let $D$ be a finite-rank operator on $\mathcal{H}$. For any $x \neq y$,

$$
d_{D}(x, y)=\infty .
$$

For instance, let $D=\left|\psi_{0}\right\rangle\left\langle\psi_{0}\right|$ be the rank 1 projection in the direction of a unit vector $\psi_{0}$. Then, $\|[D, f]\|$ is the uncertainty of $f$ relative to the vector state $\psi_{0}[10$, Lemma 5.3] (see also [31]):

$$
\|[D, f]\|^{2}=\left\langle f \psi_{0}, f \psi_{0}\right\rangle-\left|\left\langle\psi_{0}, f \psi_{0}\right\rangle\right|^{2} \quad \forall f \in \mathcal{A} .
$$


It follows from Cauchy-Schwarz inequality that (8) is zero if and only if $f$ is constant or $f \psi_{0}=0$. In particular, if the support of $\psi_{0}$ is the whole $M$ (e.g., $\psi_{0}$ is a Gaussian for $M=\mathbb{R}^{d}$ ), then (8) vanishes only if $f$ is constant, although $d_{D}$ is infinite by Proposition 2.3.

More generally, given a spectral triple $(\mathcal{A}, \mathcal{H}, D)$, if the commutant of $D$ in $\mathcal{A}$ is $\mathbb{C} 1$ (unital case) or $\{0\}$ (non-unital case) we say that the seminorm $L_{D}=\|[D, \cdot]\|$ on $\mathcal{A}$ induced by the Dirac operator is Lipschitz [30], or that $D$ has trivial commutant in $\mathcal{A}$. If $\mathcal{A}$ is unital with separable norm-closure, its representation is non-degenerate and $D$ has a compact resolvent, then $d_{D}$ is finite on the space of states of $\mathcal{A}$ as soon as $L_{D}$ is Lipschitz [28, Proposition B.1]. Together with Proposition 2.2, this shows in particular that on a compact manifold the spectral distance $d_{D}$ defined by an operator $D$ with compact resolvent is finite if and only if $L_{D}$ is Lipschitz. In the light of Proposition 2.3 one may wonder if an alternative characterization of the finiteness of $d_{D}$ could be: assuming $L_{D}$ is Lipschitz, then $d_{D}$ is finite if and only $D$ has compact resolvent. So far, we have no answer to this question. Hints may come from Rieffel's characterization of the finiteness of the distance based on general properties of the semi-norm $L_{D}$ [29].

In the non-unital case the situation is more involved. Instead of a compact resolvent, one asks for condition (ii). However the latter, together with the triviality of the commutant of $D$, no longer guarantees that the distance is finite. For instance, in the Moyal plane there are states (even pure) at infinite distance from one another [7], although the Dirac operator satisfies (ii) and has a trivial commutant in $\mathcal{A}$. As well, on a locally compact manifold with $D$ the usual Dirac operator, there are states at infinite distance from one another (for instance a state whose momentum of order 1 is infinite is at infinite distance from any point). However two pure states (i.e. two points) are at finite distance as soon as $M$ is locally compact and complete. So one may wonder if the following holds: on a locally compact and complete manifold, assuming $D$ has trivial commutant, the distance $d_{D}$ between points of $M$ is finite if and only if $D$ satisfies condition (ii).

\subsection{Commutator and edge effects}

We now discuss what type of position-momentum commutation relations are associated to the regularized Dirac operators considered above. For simplicity, we focus on the case $M=\mathbb{R}$.

A way to regularize the spectrum of the Dirac operator $D=-i \mathrm{~d} / \mathrm{d} x$ of the real line is to replace it with $D_{\Lambda}=P_{\Lambda} D$, where $P_{\Lambda}$ is the spectral projection of $D$ in the interval $[-\Lambda, \Lambda]$. In the momentum representation, the position and momentum operators $\widetilde{\boldsymbol{x}}, \widetilde{\boldsymbol{p}}$ are the endormorphisms of $\mathcal{S}(\mathbb{R})$

$$
\widetilde{\boldsymbol{x}}(\psi)(p)=i \frac{\mathrm{d}}{\mathrm{d} p} \psi(p), \quad \widetilde{\boldsymbol{p}}(\psi)(p)=p \psi(p),
$$

obtained by conjugating $\boldsymbol{x}$ and $\boldsymbol{p}$ with the Fourier transform $\mathcal{F}$. The truncated momentum operator is $\tilde{\boldsymbol{p}}_{\Lambda}=\mathbb{I}_{\Lambda} \tilde{\boldsymbol{p}}$, where $\mathbb{I}_{\Lambda}:=\mathcal{F} P_{\Lambda} \mathcal{F}^{-1}$ is the operator of pointwise multiplication by the characteristic function of the interval $[-\Lambda, \Lambda]$. The range of $\widetilde{\boldsymbol{p}}_{\Lambda}$ is not in the domain of $\widetilde{\boldsymbol{x}}$ due to the non-differentiability of the characteristic function at $\pm \Lambda$, so that the commutator $\left[\widetilde{\boldsymbol{x}}, \tilde{\boldsymbol{p}}_{\Lambda}\right]$ is ill defined.

To cure this, one can define $\widetilde{\boldsymbol{x}}$ on $\mathbb{I}_{\Lambda} \mathcal{S}(\mathbb{R})$ as $\widetilde{\boldsymbol{x}}\left(\mathbb{I}_{\Lambda} \psi\right):=\mathbb{I}_{\Lambda} \widetilde{\boldsymbol{x}} \psi$ for all $\psi \in \mathcal{S}(\mathbb{R})$. This is just the derivative of $\mathbb{I}_{\Lambda} \psi$, everywhere the latter is defined, and is compactly supported hence $L^{2}$. Then

$$
\left\langle\varphi, \widetilde{\boldsymbol{x}} \widetilde{\boldsymbol{p}}_{\Lambda} \psi\right\rangle-\left\langle\varphi, \widetilde{\boldsymbol{p}}_{\Lambda} \widetilde{\boldsymbol{x}} \psi\right\rangle=i\left\langle\varphi, \mathbb{I}_{\Lambda} \psi\right\rangle \quad \forall \varphi, \psi \in \mathcal{S}(\mathbb{R}),
$$

which we interpret (in the weak sense) as the phase-space commutation relation:

$$
\left[\widetilde{\boldsymbol{x}}, \widetilde{\boldsymbol{p}}_{\Lambda}\right]=i \mathbb{I}_{\Lambda}
$$


Another approach is by defining the derivative of $\mathbb{I}_{\Lambda} \psi, \psi \in \mathcal{S}(\mathbb{R})$, in the sense of distributions. Let $\mathcal{S}^{\prime}(\mathbb{R})$ be the space of tempered distributions and recall that any $L^{2}$-function $f$ is the integral kernel of a tempered distribution $T_{f}$. As usual, we think of $\mathcal{S}(\mathbb{R})$ and $L^{2}(\mathbb{R})$ as subspaces of $\mathcal{S}^{\prime}(\mathbb{R})$ via the monomorphism $f \mapsto T_{f}$. Any $\xi \in \operatorname{End}(\mathcal{S}(\mathbb{R}))$ can be extended to an endomorphism of $\mathcal{S}^{\prime}(\mathbb{R})$ by duality. In particular, $\widetilde{\boldsymbol{x}}(T)(f):=-T(\widetilde{\boldsymbol{x}} f) \forall T \in \mathcal{S}^{\prime}(\mathbb{R}), f \in \mathcal{S}(\mathbb{R})$ is the derivative in the sense of distributions (besides the $i$ factor). As well, any bounded operator $\xi$ on $L^{2}(\mathbb{R})$ can be extended to a linear map $L^{2}(\mathbb{R}) \rightarrow \mathcal{S}^{\prime}(\mathbb{R}), f \mapsto T_{\xi f}$; this in particular applies to $\mathbb{I}_{\Lambda}$ and $\widetilde{\boldsymbol{p}}_{\Lambda}$. Integration by parts gives:

$$
\widetilde{\boldsymbol{x}}\left(T_{\widetilde{\boldsymbol{p}}_{\Lambda} f}\right)(g)-T_{\widetilde{\boldsymbol{p}}_{\Lambda} \widetilde{\boldsymbol{x}} f}(g)=-\left.i p f g\right|_{-\Lambda} ^{\Lambda}+i T_{\mathbb{I}_{\Lambda} f}(g) \quad \forall f, g \in \mathcal{S}(\mathbb{R}) .
$$

Denoting by $\hat{\delta}_{x}: \mathcal{S}(\mathbb{R}) \rightarrow \mathcal{S}^{\prime}(\mathbb{R})$ the linear map $\hat{\delta}_{x}(f)=f(x) \delta_{x}$, from (10) one obtains the identity of linear maps $\mathcal{S}(\mathbb{R}) \rightarrow \mathcal{S}^{\prime}(\mathbb{R})$ :

$$
\left[\widetilde{\boldsymbol{x}}, \widetilde{\boldsymbol{p}}_{\Lambda}\right]=i \mathbb{I}_{\Lambda}-i \Lambda\left\{\hat{\delta}_{\Lambda}+\hat{\delta}_{-\Lambda}\right\}
$$

In addition to (9) which amounts to replacing the identity operator of the CCR by the cutoff function $\mathbb{I}_{\Lambda}$, the r.h.s. of (11) takes also into account the edge contributions due to the non-differentiability of the cut-off.

Equations (9) and (11) are two ways to interpret the cut-off on momenta in terms of a modification of the CCR, based on two possible extensions of the position operator. Regularizing the momentum operator provides a first example of modification of both the CCR and the spectral distance: since $\left\|\boldsymbol{p}_{\Lambda}\right\|=\Lambda$, we know from Proposition 2.1 that there is a minimum distance, that is $d_{p_{\Lambda}}(x, y) \geq \Lambda^{-1} \forall x \neq y$. However, it is not clear how to compute the distance, or even find an upper bound. It is an open problem to understand whether it is always infinite, as in the finite-rank case, or not.

\section{Spectral distance and phase-space commutation relations}

In this section, we study other modifications of the distance on $\mathbb{R}$ coming from deformations of the CCR: first deformations associated with non-Euclidean metrics, then two approximations of the derivative on $\mathbb{R}$ by finite difference operators. These commutation relations are not implemented by self-adjoint, nor even symmetric, operators. For a study of metric aspects this is not a problem, since formula (6) makes sense also for non-selfadjoint (and not even symmetric) momentum operators $\boldsymbol{p}$.

\subsection{Non-flat metric: $[x, p]=G(x)$}

Given a (Riemannian) metric tensor $g$ on the real line, i.e. a strictly positive smooth function, the associated geodesic distance (for $x<y$ ) is

$$
d_{g}(x, y):=\int_{x}^{y} \sqrt{g(t)} \mathrm{d} t .
$$

If $G$ is a slowly increasing complex smooth function on $\mathbb{R}$ (hence, in the multiplier algebra of $\mathcal{S}(\mathbb{R})[33]$ ), the deformed momentum operator

$$
\boldsymbol{p}(f)(x):=-G(x) \partial_{x} f(x)
$$

is a well-defined endomorphism of $\mathcal{S}(\mathbb{R})$ and satisfies the commutation relation

$$
[\boldsymbol{x}, \boldsymbol{p}]=G(\boldsymbol{x}) .
$$


Proposition 3.1. Suppose $G$ is never zero, $|G|^{-1}$ is smooth and the metric (12) with $g:=|G|^{-2}$ is complete. Then the spectral distance $d_{\boldsymbol{p}}$ is the geodesic distance $d_{g}$.

Proof. The norm inequality in (6) in the present case becomes

$$
\left|f^{\prime}(t)\right| \leq \sqrt{g(t)} \quad \forall t \in \mathbb{R}
$$

which integrated gives (for $x<y$ ):

$$
|f(x)-f(y)|=\left|\int_{x}^{y} f^{\prime}(t) \mathrm{d} t\right| \leq \int_{x}^{y} \sqrt{g(t)} \mathrm{d} t=d_{g}(x, y) .
$$

Hence $d_{p}(x, y) \leq d_{g}(x, y)$

Note that the function $f_{y}(t):=d_{g}(t, y)$ satisfies $(13)$ and $f_{y}(x)-f_{y}(y)=d_{g}(x, y)$, but it is not $C_{0}^{\infty}$. To prove that the inequality (14) is saturated, one uses a sequence of $C_{0}^{\infty}$ approximations of $f_{y}(t)$ (see the proof of [11, Proposition 2.1] for details).

\section{$3.2 h$-derivative: $[x, p]=i-h p$}

The $h$-derivative of a function $f$ is defined by [15]:

$$
D_{h} f(x)=\frac{f(x+h)-f(x)}{h},
$$

where $h>0$ is a real deformation parameter, and is a bounded operator on $L^{2}(\mathbb{R})$. It has norm $\Lambda=2 h^{-1}$ and converges pointwise to the usual derivative (if $f$ is differentiable) for $h \rightarrow 0$. Let

$$
\boldsymbol{p}:=-i D_{h}=-i h^{-1}\left(U_{h}-1\right),
$$

with $U_{t}$ the translation operator

$$
\left(U_{t} f\right)(x):=f(x+t) .
$$

We obtain a linear deformation of the CCR:

$$
[\boldsymbol{x}, \boldsymbol{p}]=i-h \boldsymbol{p} .
$$

Remark 3.2. The operator in equation (15) is not symmetric. Alternatively we may consider the self-adjoint version $\boldsymbol{p}^{\prime}:=-\frac{i}{2 h}\left(U_{h}-U_{-h}\right)$. However this would make the computation of the distance more difficult. Notice also that this yields a non-polynomial deformation of the CCR, for $\left[\boldsymbol{x}, \boldsymbol{p}^{\prime}\right]=\frac{i}{2}\left(U_{h}+U_{-h}\right)$ is not a polynomial in $\boldsymbol{p}^{\prime}$.

The operator $\boldsymbol{p}$ is bounded but has no finite rank. From Proposition 2.1 we know that $d_{D_{h}}(x, y)$ is greater than $\frac{h}{2}$, but we have no information on its finiteness. We now show that two points are at finite distance if and only if they are in the same orbit for the action of $\mathbb{Z}$ generated by the translation $x \mapsto x+h$. To this aim, we need the following lemma.

Lemma 3.3. For any $f \in C_{0}^{\infty}(\mathbb{R})$, we have $\left\|\left[D_{h}, f\right]\right\|=\left\|D_{h} f\right\|_{\infty} \leq\left\|f^{\prime}\right\|_{\infty}$.

Proof. From $D_{h}=h^{-1}\left(U_{h}-1\right)$, one deduces $\left[D_{h}, f\right]=\left(D_{h} f\right) U_{h}$. Since $U_{h}$ is unitary, we get $\left\|\left[D_{h}, f\right]\right\|=\left\|D_{h} f\right\|_{\infty}=h^{-1} \sup _{x \in \mathbb{R}}|f(x+h)-f(x)|$. The inequality in the statement of the lemma then follows from $|f(x)-f(y)| \leq|x-y| \cdot\left\|f^{\prime}\right\|_{\infty}$.

Proposition 3.4. $d_{D_{h}}(x, y)=|x-y|$ if $x-y \in h \mathbb{Z}$, and is infinite otherwise. 
Proof. The distance is symmetric, so we assume $x>y$. Take $x=y+n h$ with $n \geq 1$, and define $y_{k}:=y+k h$. Lemma 3.3 yields

$$
\begin{aligned}
|f(x)-f(y)| & \leq \sum_{k=0}^{n-1}\left|f\left(y_{k}\right)-f\left(y_{k}+h\right)\right| \\
& \leq n \sup _{t \in \mathbb{R}}|f(t)-f(t+h)|=n h\left\|D_{h} f\right\|_{\infty}=n h\left\|\left[D_{h}, f\right]\right\| .
\end{aligned}
$$

Thus $d_{D_{h}}(x, y) \leq n h=|x-y|$. Now, $\left\|\left[D_{h}, f\right]\right\| \leq\left\|f^{\prime}\right\|_{\infty}$ implies the opposite inequality between the dual distance, that is $d_{D_{h}}(x, y) \geq|x-y|$. This proves the first statement.

For $n>0$, consider the smooth function vanishing at infinity:

$$
f_{n, x}(t)=n e^{-\frac{1}{n} \sqrt{1+(t-x)^{2}}} \sin ^{2} \frac{\pi(x-t)}{h} .
$$

Since the trigonometric part is $h$-periodic, $D_{h} f_{n, x}(t)=n \sin ^{2} \frac{\pi(x-t)}{h} D_{h} e^{-\frac{1}{n} \sqrt{1+(t-x)^{2}}}$. Applying Lemma 3.3 to the exponential, whose Lipschitz norm is no greater than $1 / n$, we get $\left\|\left[D_{h}, f_{n, x}\right]\right\| \leq 1$. This implies

$$
d_{D_{h}}(x, y) \geq\left|f_{n, x}(x)-f_{n, x}(y)\right|=n e^{-\frac{1}{n} \sqrt{1+(x-y)^{2}}} \sin ^{2} \frac{\pi(x-y)}{h} .
$$

If $x-y \notin h \mathbb{Z}$, the lower bound is not zero, and goes to infinity for $n \rightarrow \infty$.

We wonder if there exists a family of states convergent to pure states and whose distance is the Euclidean one. Natural candidates are the rectangular distributions

$$
\delta_{x, \epsilon}(f)=\frac{1}{\epsilon} \int_{x-\epsilon / 2}^{x+\epsilon / 2} f(t) \mathrm{d} t, \quad \epsilon>0,
$$

since the net $\left\{\delta_{x, \epsilon}\right\}_{\epsilon>0}$ is weakly convergent to $\delta_{x}$. For a fixed $\epsilon$, call $\mathbb{R}_{\epsilon}=\left\{\delta_{x, \epsilon}\right\}_{x \in \mathbb{R}}$

Proposition 3.5. $\left(\mathbb{R}_{\epsilon}, d_{D_{h}}\right)$ is a metric space isometric to the Euclidean real line iff $\epsilon$ is a multiple of $h$ :

$$
\begin{aligned}
& \text { if } \epsilon \in h \mathbb{Z}^{+} \quad \text { then } \quad d_{D_{h}}\left(\delta_{x, \epsilon}, \delta_{y, \epsilon}\right)=|x-y| \quad \forall x, y \in \mathbb{R} \text {, } \\
& \text { if } \epsilon \notin h \mathbb{Z}^{+} \quad \text { then } \quad d_{D_{h}}\left(\delta_{x, \epsilon}, \delta_{y, \epsilon}\right)= \begin{cases}|x-y| & \text { if } x-y \in h \mathbb{Z}, \\
\infty & \text { otherwise. }\end{cases}
\end{aligned}
$$

Proof. Assume that $x>y$. From the inequality in Lemma 3.3, it follows

$$
d_{D_{h}}\left(\delta_{x, \epsilon}, \delta_{y, \epsilon}\right) \geq d_{D}\left(\delta_{x, \epsilon}, \delta_{y, \epsilon}\right)=|x-y|,
$$

where $D$ is the usual derivative and the last equality comes, e.g., from [11, Proposition 3.2].

Let $\epsilon \in h \mathbb{Z}^{+}$and $n$ be the integer part of $(x-y) / \epsilon$, that is $x-y-\epsilon<n \epsilon \leq x-y$. Then

$$
\begin{aligned}
\epsilon \delta_{x, \epsilon}(f) & =\int_{n \epsilon-\epsilon / 2}^{n \epsilon+\epsilon / 2} f(t+y) \mathrm{d} t+\int_{n \epsilon+\epsilon / 2}^{x-y+\epsilon / 2} f(t+y) \mathrm{d} t-\int_{n \epsilon-\epsilon / 2}^{x-y-\epsilon / 2} f(t+y) \mathrm{d} t \\
& =\int_{-\epsilon / 2}^{\epsilon / 2} f(t+y+n \epsilon) \mathrm{d} t+\int_{n \epsilon-\epsilon / 2}^{x-y-\epsilon / 2}(f(t+y+\epsilon)-f(t+y)) \mathrm{d} t .
\end{aligned}
$$

For any $f$ with $\left\|\left[D_{h}, f\right]\right\| \leq 1$,

$$
\epsilon\left(\delta_{x, \epsilon}-\delta_{y, \epsilon}\right)(f)=\int_{-\epsilon / 2}^{\epsilon / 2}(f(t+y+n \epsilon)-f(t+y)) \mathrm{d} t
$$




$$
\begin{aligned}
& +\int_{n \epsilon-\epsilon / 2}^{x-y-\epsilon / 2}(f(t+y+\epsilon)-f(t+y)) \mathrm{d} t \\
\leq & n \epsilon \int_{-\epsilon / 2}^{\epsilon / 2} \mathrm{~d} t+\epsilon \int_{n \epsilon-\epsilon / 2}^{x-y-\epsilon / 2} \mathrm{~d} t=n \epsilon^{2}+\epsilon(x-y-n \epsilon)=\epsilon(x-y),
\end{aligned}
$$

where we use $f(t+y+n \epsilon)-f(t+y) \leq n \epsilon$, that follows from the norm condition. Hence $d_{D_{h}}\left(\delta_{x, \epsilon}, \delta_{y, \epsilon}\right) \leq|x-y|$. Equation (18a) follows from (19).

Similarly, for any $\epsilon$ and any $x=y+n h$ with $n \geq 1$, one has

$$
\delta_{x, \epsilon}(f)-\delta_{y, \epsilon}(f)=\frac{1}{\epsilon} \int_{-\epsilon / 2}^{\epsilon / 2}(f(t+x)-f(t+y)) \mathrm{d} t \leq \frac{n h}{\epsilon} \int_{-\epsilon / 2}^{\epsilon / 2} 1 \mathrm{~d} t=n h=|x-y| .
$$

Hence the first equation in (18b). The second one is obtained by considering the following function. Let $f_{\infty, x}(t):=\sin ^{2} \frac{\pi(t-x)}{h}, e_{n}(t):=e^{-\frac{1}{n} \sqrt{1+t^{2}}}$ and $f_{K, n, x}(t)=K e_{n}(t-x) f_{\infty, x}(t)$, with $n \in \mathbb{Z}^{+}$and $K>0$. Similarly to $(16),\left\|\left[D_{h}, f_{K, n, x}\right]\right\| \leq 1$ for all $K<n$. Since $e_{n}(t-y)$ is uniformly convergent to 1 in the interval $[x-\epsilon / 2, x+\epsilon / 2]$, then

$$
\lim _{n \rightarrow \infty} \delta_{x, \epsilon}\left(f_{K, n, y}\right)=K \delta_{x, \epsilon}\left(f_{\infty, y}\right)=\frac{K}{2 \epsilon}\left[t-\frac{h}{2 \pi} \sin \frac{2 \pi t}{h}\right]_{x-y-\frac{\epsilon}{2}}^{x-y+\frac{\epsilon}{2}} .
$$

Therefore, using some trigonometric identities:

$$
d_{D_{h}}\left(\delta_{x, \epsilon}, \delta_{y, \epsilon}\right) \geq \lim _{n \rightarrow \infty}\left\{\delta_{x, \epsilon}\left(f_{K, n, y}\right)-\delta_{y, \epsilon}\left(f_{K, n, y}\right)\right\}=\frac{K h}{\pi \epsilon} \sin \frac{\pi \epsilon}{h} \sin ^{2} \frac{\pi(x-y)}{h},
$$

for all $K>0$. If $\epsilon \notin h \mathbb{Z}^{+}$and $x-y \notin h \mathbb{Z}$, the right hand side goes to infinity for $K \rightarrow \infty$.

Although the maximal resolution between points is of order $\left\|D_{h}\right\|^{-1} \sim h$, this result shows that the Euclidean distance is recovered by considering non-pure states, like rectangular distributions with width $\epsilon=h$.

Remark 3.6. One may wonder what remains true for states that are more general than (17). Given a positive integrable function $\psi$ supported in the interval $[-\epsilon, \epsilon]$ and normalized to 1 , consider the corresponding family of states (for $x \in \mathbb{R}$ ):

$$
\Psi_{x, \epsilon}(f)=\int_{-\epsilon / 2}^{\epsilon / 2} \psi(t) f(t+x) \mathrm{d} t .
$$

The inequality (20) is still valid. Thus $d_{D_{h}}\left(\Psi_{x, \epsilon}, \Psi_{y, \epsilon}\right)=|x-y|$ if $x-y \in h \mathbb{Z}$ (for any value of $\epsilon$ ). For $x-y \notin h \mathbb{Z}$, computing the distance is an open problem.

\section{$3.3 q$-derivative: $x p-q p x=i$}

Another well-known "approximation" of the derivative is the $q$-derivative [19]

$$
D_{q} f(x)=\frac{f(x)-f(q x)}{(1-q) x} \quad \forall x \neq 0 .
$$

Here $0<q<1$ is a deformation parameter. The $q$-derivative is extended by continuity at $x=0: D_{q} f(0)=f^{\prime}(0)$ (provided the r.h.s. is well-defined). Setting $\boldsymbol{p}=-i D_{q}$, one obtains the deformation of the CCR:

$$
\boldsymbol{x p}-q \boldsymbol{p} \boldsymbol{x}=i .
$$


This $q$-deformed phase-space was studied in [13]. The momentum operator is not symmetric, and a discussion on how to deform the conjugation operation in a way that is consistent with the commutation relations is in [13].

Because of the behavior at $x=0, D_{q}$ is not a bounded operator, so one cannot expect a minimum length. We show that the distance between pure states is always finite, and bounded by the "French railway metric": $d_{\mathrm{SNCF}}(x, y):=|x|+|y|$ for any $x \neq y, d_{\mathrm{SNCF}}(x, x)=0$.

Lemma 3.7. For any $f \in C_{0}^{\infty}(\mathbb{R})$, we have $q^{\frac{1}{2}}\left\|\left[D_{q}, f\right]\right\|=\left\|D_{q} f\right\|_{\infty} \leq\left\|f^{\prime}\right\|_{\infty}$.

Proof. From the $q$-analogue of the Leibniz rule, we get $\left[D_{q}, f\right] \psi(x)=\psi(q x) D_{q} f(x)$ for all $x \in \mathbb{R}$ (including $x=0$ ). For $\rho>0$, let $T_{\rho}$ be the unitary operator defined by $T_{\rho} \psi(x)=\rho^{\frac{1}{2}} \psi(\rho x)$. Then $\left[D_{q}, f\right]=q^{-\frac{1}{2}}\left(D_{q} f\right) T_{q}$, and

$$
\left[D_{q}, f\right]^{*}\left[D_{q}, f\right]=q^{-1} T_{q}^{*}\left|D_{q} f\right|^{2} T_{q} .
$$

Hence $\left\|\left[D_{q}, f\right]\right\|^{2}=q^{-1}\left\|D_{q} f\right\|_{\infty}^{2}$. The result follows from $|f(x)-f(y)| \leq|x-y| \cdot\left\|f^{\prime}\right\|_{\infty}$.

Proposition 3.8. For any $x, y$,

$$
q^{\frac{1}{2}}|x-y| \leq d_{D_{q}}(x, y) \leq q^{\frac{1}{2}} d_{\mathrm{SNCF}}(x, y) .
$$

If further $x$ and $y$ are in the same orbit of $q^{\mathbb{Z}}$, then

$$
d_{D_{q}}(x, y)=q^{\frac{1}{2}}|x-y|
$$

Proof. Assume $x>y$. By Lemma 3.7 we get $d_{D_{q}}(x, y) \geq q^{\frac{1}{2}}|x-y|$. If $y=q^{n} x$, with $n \geq 1$, then

$$
\begin{aligned}
|f(x)-f(y)| & \leq \sum_{k=0}^{n-1}\left|f\left(x_{k}\right)-f\left(x_{k+1}\right)\right| \\
& \leq\left\|D_{q} f\right\|_{\infty} \sum_{k=0}^{n-1}(1-q)\left|x_{k}\right|=\left\|D_{q} f\right\|_{\infty}\left(1-q^{n}\right)|x|=q^{\frac{1}{2}}\left\|\left[D_{q}, f\right]\right\||x-y|,
\end{aligned}
$$

where $x_{k}:=q^{k} x$. Thus $d_{D_{q}}(x, y) \leq q^{\frac{1}{2}}|x-y|$, which proves the first equation in (21) and (22).

For $n \rightarrow \infty, y=q^{n} x \rightarrow 0$ and we get $|f(x)-f(0)| \leq q^{\frac{1}{2}}\left\|\left[D_{q}, f\right]\right\||x|$ for all $x \in \mathbb{R}$. So, for any $x, y \in \mathbb{R}:$

$$
|f(x)-f(y)| \leq|f(x)-f(0)|+|f(0)-f(y)| \leq q^{\frac{1}{2}}\left\|\left[D_{q}, f\right]\right\| d_{\mathrm{SNCF}}(x, y),
$$

which gives the upper bound in (21).

Note that if $x, y$ are small, $d_{D_{q}}(x, y)$ can be as small as we want: as expected, there is no minimum length. The geometrical reason why the distance is always finite is that all the orbits of $q^{\mathbb{Z}}$ have a common accumulation point, given by $x=0$.

\subsection{Metrics on the momentum space}

We have shown by examples how a modification of the CCR implies a modification of the metric structure of the position space. To avoid confusion, let us stress that we are not dealing with a "noncommutative space" whose coordinates $x^{\mu}, x^{\nu}$ do not commute (the metric aspect of such spaces has been studied, e.g., in [7, 23, 24, 25]). Here the point of view is the one of quantum 
mechanics, and the noncommutativity is between the coordinate operators of the position space $(x$-space) and those of the momentum space ( $p$-space).

Since we remain at a formal level and are not considering any specific physical example, the distinction between the $x$ and $p$ spaces is mostly a matter of convention. One may as well use the CCR and its deformations to define an extended metric on the $p$-space:

$$
d_{\boldsymbol{x}}(q, p):=\sup _{f \in C_{0}^{\infty}\left(\mathbb{R}^{d}, \mathbb{R}\right)}\{f(q)-f(p):\|[\boldsymbol{x}, f(\boldsymbol{p})]\| \leq 1\} .
$$

When the commutation relation is symmetric in $\boldsymbol{x}-\boldsymbol{p}$ (as for the undeformed CCR), one gets the same metric. But this is not always the case: for the $q$-derivative of Section 3.7, one also has to make the change $q \rightarrow q^{-1}$. In the $h$-derivative example $[\boldsymbol{x}, \boldsymbol{p}]=i-h \boldsymbol{p}$ of Section 3.2 , the $p$ metric (23) is the same as the $x$-metric (6) coming from $[\boldsymbol{x}, \boldsymbol{p}]=-i+h \boldsymbol{x}$, which is a example of non-flat deformation of the $x$-space as studied in Section 3.1.

Another interesting example is the $x$-metric for a non-flat deformation of the $p$-space (equivalently: the $p$-metric for a non-flat deformation of the $x$-space):

$$
[\boldsymbol{x}, \boldsymbol{p}]=G(\boldsymbol{p}),
$$

where $G$ is some well-behaved function. For instance Kempf, Mangano and Mann [17, 18] studied the quadratic deformation

$$
[\boldsymbol{x}, \boldsymbol{p}]=i\left(1+\ell^{2} \boldsymbol{p}^{2}\right)
$$

where $\ell>0$ has the dimension of a length. Formally one can obtain this commutation relation from the operator

$$
\boldsymbol{p}=-i \ell^{-1} \frac{U_{\ell}-U_{-\ell}}{U_{\ell}+U_{-\ell}}
$$

and link the corresponding distance to the one of the $h$-derivative $D_{h}$ for $h=2 \ell$, noticing that

$$
D_{2 \ell} f=i\left(U_{2 \ell}+1\right)[\boldsymbol{p}, f]\left(U_{-2 \ell}+1\right) .
$$

However, one has to be cautious with the domain of $\left(U_{\ell}+U_{-\ell}\right)^{-1}$.

Notice that curved momentum spaces have recently been under investigation, since they are expected to be at the heart of a new view on relativity [2].

\section{Fat points and optimal transport on the circle}

In Section 3.2 we show how to recover the Euclidean distance from a deformed CCR using, instead of points, non-pure states given by rectangular distributions. In this section, we illustrate another interest for such "fat points" by computing the distance between rectangular distributions on the circle. This gives an example where the distance between two translated states is neither the amplitude of translation (as in the Euclidean space [11, Proposition 3.2]), nor the geodesic distance of $S^{1}$. Furthermore, this result may have an interest on its own, independently of noncommutative geometry, since as far as we know there are few examples of explicit computation of the Wasserstein distance on the circle (discrete distributions have been recently studied in [27]).

Let $\mathcal{A}=C^{\infty}\left(S^{1}, \mathbb{R}\right)$ and $D=-i \frac{\mathrm{d}}{\mathrm{d} x}$ the usual Dirac operator on the circle (we think of functions on $S^{1}$ as $2 \pi$-periodic functions on $\mathbb{R}$ ). Consider the compactly supported distribution

$$
\delta_{x, \epsilon}(f):=\int_{-\epsilon}^{\epsilon} f(t+x) \mathrm{d} \mu_{t},
$$


where $0<\epsilon<\pi$ and $\mathrm{d} \mu_{t}$ is any distribution with support in $[-\epsilon, \epsilon]$ normalized to 1 . It is enough to compute the distance for $y=0$ and $0<x \leq \pi$ since the distance is translation invariant and symmetric [10, Lemma 5.9].

Proposition 4.1. For $0<x \leq \pi-2 \epsilon$ one has

$$
d_{D}\left(\delta_{0, \epsilon}, \delta_{x, \epsilon}\right)=x .
$$

For $\mathrm{d} \mu_{t}=\frac{1}{2 \epsilon} \chi_{[-\epsilon, \epsilon]}(t) \mathrm{d} t$ the rectangular distribution and $\pi-2 \epsilon \leq x \leq \pi$, one has (Fig. 1(a))

$$
d_{D}\left(\delta_{0, \epsilon}, \delta_{x, \epsilon}\right)=\frac{1}{4 \epsilon}\left(-x^{2}+2 \pi x-(\pi-2 \epsilon)^{2}\right) .
$$

Proof. Consider the 1-Lipschitz function:

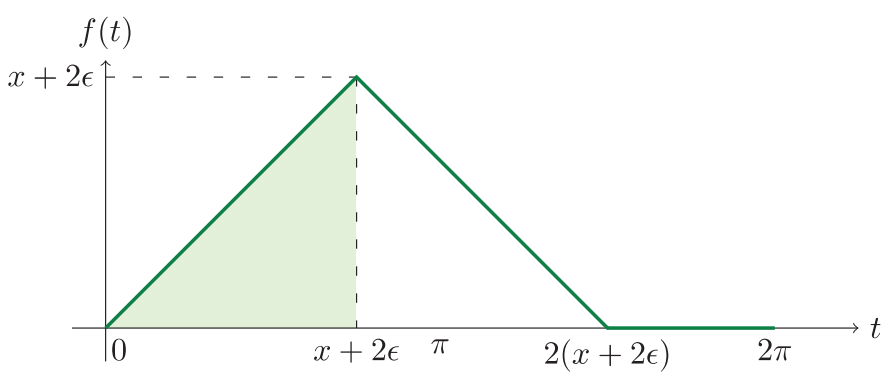

With the replacement $f(x) \rightarrow f(x+\epsilon)$, we get for $0<x \leq \pi-2 \epsilon$ :

$$
d_{D}\left(\delta_{0, \epsilon}, \delta_{x, \epsilon}\right) \geq \int_{0}^{2 \epsilon}\{f(t+x)-f(t)\} \mathrm{d} \mu_{t-\epsilon}=\int_{0}^{2 \epsilon}(t+x-t) \mathrm{d} \mu_{t-\epsilon}=x \int_{-\epsilon}^{\epsilon} \mathrm{d} \mu_{t}=x .
$$

On the other hand for any periodic $f$ with $\|[D, f]\|_{\infty}=\sup _{t}\left|f^{\prime}(t)\right| \leq 1$ one has:

$$
\left|\delta_{0, \epsilon}-\delta_{x, \epsilon}\right| \leq \int_{-\epsilon}^{\epsilon}|f(t+x)-f(x)| \mathrm{d} \mu_{t} \leq\left\|f^{\prime}\right\|_{\infty} \cdot x=x
$$

Hence the opposite inequality, that implies (24).

For $\pi-2 \epsilon \leq x \leq \pi$ and for rectangular distributions, the following function

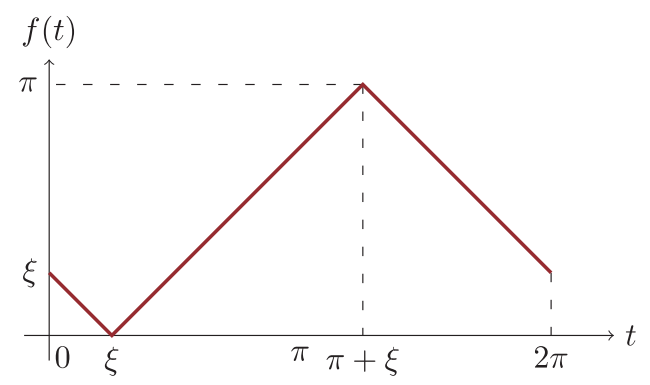

yields

$$
\begin{aligned}
2 \epsilon d_{D}\left(\delta_{0, \epsilon}, \delta_{x, \epsilon}\right) & \geq \int_{0}^{2 \epsilon}\{f(t+x)-f(t)\} \mathrm{d} t \\
& =\int_{0}^{\xi}\{f(t+\pi+\xi)-f(t)\} \mathrm{d} t+\int_{\xi}^{2 \epsilon}\{f(t+x-\xi)-f(t)\} \mathrm{d} x \\
& \geq \int_{0}^{\xi}\{(\pi-t)-(\xi-t)\} \mathrm{d} t+\int_{\xi}^{2 \epsilon}\{(t+x-2 \xi)-(t-\xi)\} \mathrm{d} t \\
& =\{(\pi-\xi) \xi+(x-\xi)(2 \epsilon-\xi)\}=\frac{1}{2}\left(-x^{2}+2 \pi x-(\pi-2 \epsilon)^{2}\right),
\end{aligned}
$$


where $\xi:=\frac{1}{2}(x+2 \epsilon-\pi)$. On the other hand, for any 1-Lipschitz function $f$ :

$$
\begin{aligned}
& f(t+\pi+\xi)-f(t) \leq d_{\mathrm{geo}}(0, \pi+\xi)=2 \pi-(\pi+\xi)=\pi-\xi \\
& f(t+x-\xi)-f(t) \leq d_{\mathrm{geo}}(0, x-\xi)=x-\xi
\end{aligned}
$$

where in the first equation we noticed that $\pi \leq \pi+\xi \leq 2 \pi$. Therefore

$$
\begin{aligned}
d_{D}\left(\delta_{0, \epsilon}, \delta_{x, \epsilon}\right) & \leq \frac{1}{2 \epsilon}\left(\int_{0}^{\xi}(\pi-\xi) \mathrm{d} t+\int_{\xi}^{2 \epsilon}(x-\xi) \mathrm{d} t\right) \\
& =\frac{1}{2 \epsilon}\{(\pi-\xi) \xi+(x-\xi)(2 \epsilon-\xi)\}=\frac{1}{4 \epsilon}\left(-x^{2}+2 \pi x-(\pi-2 \epsilon)^{2}\right) .
\end{aligned}
$$

Hence the inequality is actually an equality.

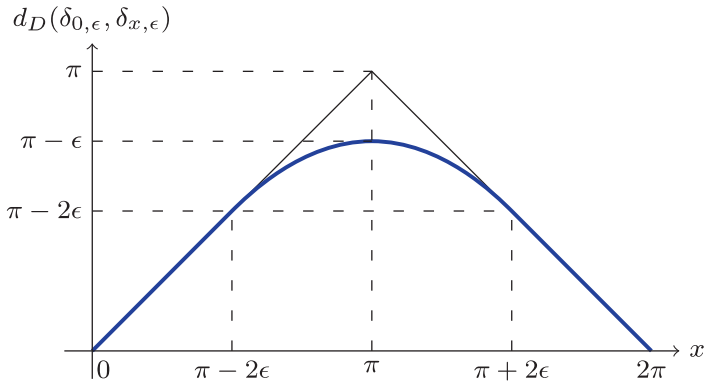

(a)

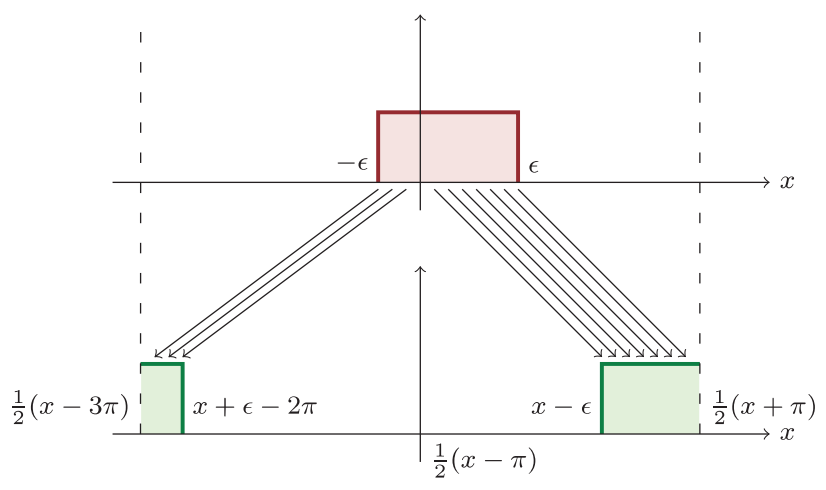

(b)

Figure 1. The distance for rectangular distributions (a). The optimal transport map for rectangular distributions (b).

When $x+2 \epsilon>\pi$ the distance is less than the geodesic one (Fig. 1(a)) because one can optimize the transport by moving part of the distribution to the left and part to the right along the circle (see Fig. 1(b)). As stressed in [6], computing the Wasserstein distance on the circle amounts to cutting the circle at a well chosen point and then computing the same distance on the real line. This cutting point is explicitly given in [6] for discrete distributions. For rectangular distributions, one sees from the proof of Proposition 4.1 that the cutting point has coordinate $\frac{1}{2}(x-\pi)$ as in Fig. 1(b).

The distance is also smoother than the Euclidean one (not at 0 though). Interestingly the same phenomenon appears in a totally different context (covariant Dirac operator on a $U(n)$ bundle on the circle $[21,22])$.

\section{Acknowledgements}

F.L. is partially supported by CUR Generalitat de Catalunya under project FPA2010-20807. F.D. and F.L. were partially supported by UniNA and Compagnia di San Paolo under the grant "Programma STAR 2013".

\section{References}

[1] Amati D., Ciafaloni M., Veneziano G., Can spacetime be probed below the string size?, Phys. Lett. B 216 (1989), 41-47.

[2] Amelino-Camelia G., Freidel L., Kowalski-Glikman J., Smolin L., The principle of relative locality, Phys. Rev. D 84 (2011), 084010, 13 pages, arXiv:1101.0931. 
[3] Amelino-Camelia G., Lukierski J., Nowicki A., $\kappa$-deformed covariant phase space and quantum-gravity uncertainty relations, Phys. Atomic Nuclei 61 (1998), 1811-1815, hep-th/9706031.

[4] Ardalan F., Arfaei H., Ghasemkhani M., Sadooghi N., Gauge invariant cutoff QED, Phys. Scr. 87 (2013), 035101, 12 pages, arXiv:1108.3215.

[5] Bronstein M., Quantum theory of weak gravitational fields, Gen. Relativity Gravitation 44 (2012), $267-283$.

[6] Cabrelli C.A., Molter U.M., The Kantorovich metric for probability measures on the circle, J. Comput. Appl. Math. 57 (1995), 345-361.

[7] Cagnache E., D'Andrea F., Martinetti P., Wallet J.C., The spectral distance in the Moyal plane, J. Geom. Phys. 61 (2011), 1881-1897, arXiv:0912.0906.

[8] Connes A., Noncommutative geometry, Academic Press, Inc., San Diego, CA, 1994.

[9] Connes A., Marcolli M., Noncommutative geometry, quantum fields and motives, American Mathematical Society Colloquium Publications, Vol. 55, Amer. Math. Soc., Providence, RI, 2008.

[10] D'Andrea F., Lizzi F., Martinetti P., Spectral geometry with a cut-off: topological and metric aspects, J. Geom. Phys. 82 (2014), 18-45, arXiv:1305.2605.

[11] D'Andrea F., Martinetti P., A view on optimal transport from noncommutative geometry, SIGMA 6 (2010), 057, 24 pages, arXiv:0906.1267.

[12] Doplicher S., Fredenhagen K., Roberts J.E., The quantum structure of spacetime at the Planck scale and quantum fields, Comm. Math. Phys. 172 (1995), 187-220, hep-th/0303037.

[13] Fichtmüller M., Lorek A., Wess J., q-deformed phase space and its lattice structure, Z. Phys. C 71 (1996), 533-537, hep-th/9511106.

[14] Gross D.J., Mende P.F., String theory beyond the Planck scale, Nuclear Phys. B 303 (1988), $407-454$.

[15] Kac V., Cheung P., Quantum calculus, Universitext, Springer-Verlag, New York, 2002.

[16] Kadison R.V., Liu Z., The Heisenberg relation - mathematical formulations, SIGMA 10 (2014), 009, 40 pages, arXiv:1401.6507.

[17] Kempf A., Mangano G., Minimal length uncertainty relation and ultraviolet regularisation, Phys. Rev. D 55 (1997), 7909-7920, hep-th/9612084.

[18] Kempf A., Mangano G., Mann R.B., Hilbert space representation of the minimal length uncertainty relation, Phys. Rev. D 52 (1995), 1108-1118, hep-th/9412167.

[19] Koornwinder T.H., q-Special functions, an overview, math.CA/0511148.

[20] Lizzi F., Vitale P., Gauge and Poincaré invariant regularization and Hopf symmetries, Modern Phys. Lett. A 27 (2012), 1250097, 15 pages, arXiv:1202.1190.

[21] Martinetti P., Carnot-Carathéodory metric and gauge fluctuation in noncommutative geometry, Comm. Math. Phys. 265 (2006), 585-616, hep-th/0506147.

[22] Martinetti P., Spectral distance on the circle, J. Funct. Anal. 255 (2008), 1575-1612, math.OA/0703586.

[23] Martinetti P., Mercati F., Tomassini L., Minimal length in quantum space and integrations of the line element in noncommutative geometry, Rev. Math. Phys. 24 (2012), 1250010, 36 pages, arXiv:1106.0261.

[24] Martinetti P., Tomassini L., Length and distance on a quantum space, PoS Proc. Sci. (2011), PoS(CORFU2011), 042, 30 pages, arXiv:1205.2908.

[25] Martinetti P., Tomassini L., Noncommutative geometry of the Moyal plane: translation isometries, Connes' distance on coherent states, Pythagoras equality, Comm. Math. Phys. 323 (2013), 107-141, arXiv:1110.6164.

[26] Piacitelli G., Quantum spacetime: a disambiguation, SIGMA 6 (2010), 073, 43 pages, arXiv:1004.5261.

[27] Rabin J., Delon J., Gousseau Y., Transportation distances on the circle, J. Math. Imaging Vision 41 (2011), 147-167, arXiv:0906.5499.

[28] Rennie A., Varilly J.C., Reconstruction of manifolds in noncommutative geometry, math.OA/0610418.

[29] Rieffel M.A., Metrics on states from actions of compact groups, Doc. Math. 3 (1998), 215-229, math.OA/9807084.

[30] Rieffel M.A., Metrics on state spaces, Doc. Math. 4 (1999), 559-600, math.OA/9906151.

[31] Rieffel M.A., Standard deviation is a strongly Leibniz seminorm, New York J. Math. 20 (2014), 35-56, arXiv:1208.4072.

[32] Rovelli C., Smolin L., Discreteness of area and volume in quantum gravity, Nuclear Phys. B 442 (1995), 593-619, gr-qc/9411005.

[33] Voigt C., Bornological quantum groups, Pacific J. Math. 235 (2008), 93-135, math.QA/0511195. 